Laser Chem. 1988, Vol. 8, pp. 49-59

Photocopying permitted by license only

(C) 1988 Harwood Academic Publishers GmbH

Reprints available directly from the Publisher

Printed in the United Kingdom

\title{
Effect of Various Anions and Zwitterions on the Lasing Properties of a Photostable Cationic Laser Dye
}

JOEL M. KAUFFMAN

Chemistry Department, Philadelphia College of Pharmacy and Science, Philadelphia, Pennsy/vania 19104, USA

\author{
JAMES H. BENTLEY \\ US Army Missile Command, Redstone Arsenal, Huntsville, Alabama \\ 35898, USA
}

(Received July 24, 1987; in final form Sept. 3, 1987)

An unusually photostable cationic laser dye first prepared by quaternization of the free base 4PyMPO (Figure 1) with methyl p-toluenesulfonate unexpectedly had, when flashlamp pumped in methanol, half the energy output of four other $\mathrm{N}$-methyl quaternary salts: the methanesulfonate, the trifluoromethanesulfonate, the perchlorate, and the tetrafluoroborate. When four homologous zwitterions (Figure 2) prepared from 4PyMPO were tested in methanol, the output energies ranged from 1.4 to 2.5 times that of the p-toluenesulfonate, and up to 1.3 times that of the salt with the highest output. When flashlamp pumped in water, the zwitterions showed 1.5 to 2.0 times the output energy of the p-toluenesulfonate in water. Many of the dyes displayed laser output curves that were split in the middle, so that lasing energy appeared on either side of a dark valley. Among the zwitterions, a regular shifting from red to blue was observed as the carbon chain length was increased.

KEY WORDS: Laser dyes, flashlamp pumped, quaternary salts, zwitterions.

\section{INTRODUCTION}

When one considers the use of a laser dye, some of the parameters of interest are lasing wavelength, energy conversion efficiency, solu- 
bility, and photochemical lifetime. When the fluorophore of the dye is a quaternary ammonium salt, then the counterion is another variable of interest.

It has been known for a long time ${ }^{1}$ that the fluorescence of dyes is quenched by certain anions, decreasing in the order: iodide $>$ thiocyanate $>$ bromide $>$ chloride $>$ perchlorate. This order suggests that the excited state of the dye is quenched by a charge-transfer interaction. The superiority of perchlorate to chloride has been demonstrated for a coumarin-type laser dye, and for Rhodamine $6 \mathrm{G}$, for which the fluorescence quantum efficiency (FQE) of the iodide and the perchlorate in ethanol were identical and very high, but only the perchlorate had an equally high $\mathrm{FQE}$ in chloroform, the iodide being almost completely quenched. Apparently, the dye salts are fully dissociated in the hydrogen-bonding solvent ethanol, but exist as tight ion pairs in chloroform, immediately available for a reaction. ${ }^{1}$

Established cationic laser dyes such as Rhodamine $6 \mathrm{G}$, whose counterion is chloride ${ }^{2}$ have shown improvements in lifetime up to $40 \%$ when the counterion is perchlorate. ${ }^{3}$ Rhodamine $\mathrm{B}$, also a chloride salt, has less energy output than Sulforhodamine B, a sulfonate zwitterion, ${ }^{4}$ at least in certain solvents. Whether the same is true of Rhodamine $640 \mathrm{vs}$. Sulforhodamine 640 is not clear. The counterions also affect the solubility of the dyes. The impact sensitivity of aromatic amine perchlorates has been well-documented, ${ }^{5}$ and it would be advantageous to replace such a potentially dangerous anion with a more shock-resistant one such as tetrafluoroborate, which has been suggested because its sizes and charge densities are similar to those of perchlorate. ${ }^{6}$

For comparison of anions without complications related to decomposition of the cation, a readily available free base was needed which was quaternizable to photostable laser dyes of usable solubility; 2-(4-pyridyl)-5-(4-methoxy $p$ henyl)oxazole (4PyMPO-MePTs, Figure 1) was reported to be a laser dye of moderate output and exceptional lifetime when flashlamp pumped, ${ }^{7}$ and was found to be very soluble in hydrogen-bonding solvents. We intended to improve the fluorophore, but sought first to specify the suitability of a variety of counterions as defined by the level of laser energy output in methanol and ease of synthesis. Only anions of low nucleophilicity were chosenthose with a single negative charge dispersed among four electronegative atoms. In this work the effect of various counterions and zwitter- 


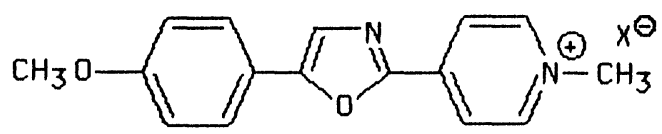

\begin{tabular}{|c|c|c|}
\hline Code & $x$ & Neme of $x$ \\
\hline 4PyMPO-MePTs & $A_{3}-\{1\}$ & $\begin{array}{l}\mathrm{p} \text {-toluenesulfonote } \\
\text { or tosylate }\end{array}$ \\
\hline 4PyMPO-MeMs & $\mathrm{CH}_{3}-\mathrm{SO}_{3}$ & $\begin{array}{l}\text { methenesulfonote } \\
\text { or mesylete }\end{array}$ \\
\hline АPyMPO-MeTf & $\mathrm{CF}_{3}-\mathrm{SO}_{3}$ & $\begin{array}{l}\text { trifluoromethene- } \\
\text { sulfongte } \\
\text { or triflete }\end{array}$ \\
\hline$\triangle \mathrm{PyMPO}-\mathrm{MeClO}_{4}$ & $\mathrm{ClO}_{4}$ & perchlorete \\
\hline$\triangle P$ yMPO-MeBF ${ }_{4}$ & $\mathrm{BF}_{4}$ & tetrafluoroborate \\
\hline
\end{tabular}

Figure 1 Structures of cationic laser dyes which are salts.

ions on energy output and lasing wavelength with flashlamp pumping was investigated for quaternary salts of $4 \mathrm{PyMPO}$ in both methanol and water.

\section{EXPERIMENTAL}

The free base used as an intermediate for all the dyes, 4PyMPO, was prepared by the method employed for the desmethoxy compound, 4 PyPO, by Ott et al. ${ }^{8}$ Synthetic details were provided for 4PyMPO by R. A. Hollins. ${ }^{9}$ Quaternizations were carried out with an excess of alkylating agent in refluxing toluene. The perchlorate and the tetrafluoroborate were prepared by addition of the appropriate acid to a solution of the tosylate in water (see Ref. 8). Where sultones were not available to make zwitterions, the quaternization was carried out by heating 4PyMPO in the appropriate alkylene dibromide at $85^{\circ}$ overnight to make the $\mathrm{N}$-bromoalkyl derivative,${ }^{10}$ and these were converted to the sulfo zwitterion by reaction with sodium sulfite. ${ }^{10}$ All compounds had infrared and proton magnetic resonance spectra in 
agreement with theory. Thin-layer chromatography on the lead compound and two of the zwitterions (MK6F silica, trifluoracetic acid) gave a single spot (with no tailing) which displayed the characteristic yellow fluorescence of these dyes under 255 or $365 \mathrm{~nm}$ ultraviolet light.

Dye solutions were made with Aldrich spectrophotometric or HLPC grade methanol which was distilled from sodium borohydride, or with deionized water.

Ultraviolet absorption data were determined with a Shimadzu UV 260, and fluorescence with a Perkin-Elmer MPF-66.

All lasing tests were performed with a Phase-R DL-1200V coaxial laser with no filtering of flashlamp light. Dye output energy was measured with a Scientech 365 power and energy meter. Laser line spectra were determined with a Tracor Northern TN-1710 multichannel analyzer fed by a Jarrell-Ash Model 82-020 monochrometer. The multichannel analyzer detector was a Tracor Northern model TN1223-31. Each reported energy and standard deviation was the result of 15 consecutive shots with an input energy of $20 \mathrm{keV}$. The positions of centroids of lasing were estimated by visual inspection of Polaroid photographs of oscilloscope traces.

\section{DISCUSSION AND RESULTS}

\section{Chemistry}

Methyl methanesulfonate reacted with the base 4PyMPO (Figure 1) to give 4PyMPO-MeMs more rapidly and in higher yield than methyl p-toluenesulfonate gave 4PyMPO-MePTs. Methyl trifluoromethanesulfonate reacted still more rapidly to give 4PyMPO-MeTf. The metathesis reactions between 4PyMPO-MePTs and perchloric or tetrafluoroboric acid in water were almost instantaneous, with precipitation of the much less soluble $4 \mathrm{PyMPO}-\mathrm{MeClO}_{4}$ and $4 \mathrm{PyMPO}-\mathrm{MeBF}_{4}$. Propanesultone reacted with 4 PyMPO much faster than butanesultone. The resulting 4PyMPO-SPrZ (Figure 2) was less soluble in water or methanol than the 4PyMPO-SBZ, and all the zwitterions were less soluble than any of the salts. All were sufficiently soluble in methanol for laser use.

While aligning and flushing our laser system, we noticed that fluorescence was observed from one lot of supposedly clean methanol 


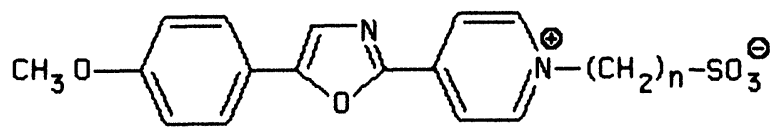

\begin{tabular}{llc} 
Code & $n$ & Name of N-Alkyl Group \\
\hline 4PyMPO-SEZ & 2 & 2-sulfoethyl \\
4PyMPO-SPrZ & 3 & 3-sulfopropyl \\
4PyMPO-SBZ & 4 & 4-sulfobutyl \\
4PyMPO-SPeZ & 5 & 5-sulfopentyl
\end{tabular}

Figure 2 Structures of cationic laser dyes which are zwitterions.

under excitation with a hand-held long-wave UV light, while unused spectrophotometric grade methanol showed no such response. An experiment was set up where methanol was exposed to 100 flashlamp discharges at $20 \mathrm{keV}$ in the dye laser, and then compared with methanol was merely pumped through the dye laser for the same time period. The appropriate spectrometers plainly showed that a chromophore developed in the "flashed" methanol, on with an absorption peak at $525 \mathrm{~nm}$ and a fluorescence emission peak at $555 \mathrm{~nm}$. We found that the source of the chromophore was eliminated by distillation of the methanol from about $0.2 \% \mathrm{w} / \mathrm{v}$ sodium borohydride.

\section{Energy Output}

Laser energy outputs (Tables I and II) of all the salts and zwitterions were strikingly greater than that of the toluenesulfonate. Although no service lifetime tests were done, there was no systematic energy reduction which would indicate premature dye degradation as an explanation for the energy differences. Delocalization of charge in the anion would be least in the methanesulfonate and in the zwitterions, and greatest in the perchlorate; however, the perchlorate did not have the greatest output energy. If the charge-transfer interaction described by Drexhage ${ }^{1}$ is operative, one must be able to rationalize why it should be greatest for the toluenesulfonate. Perhaps van der Waals attraction between the $\pi$-bonds of benzene ring of the toluenesulfonate anion and the $\pi$-bonds of the pyridine ring, in addition to ionic attraction, overcome the ability of methanol or water to separate these ions, and the proximity of the negative charge to the pyridine nitrogen 
Table I Energy output and wavelength(s) of laser dyes related to 4PyMPO at $2.5 \times$ $10^{-4} \mathrm{M}$ in methanol when flashlamp-pumped at $20 \mathrm{KeV}$.

\begin{tabular}{|c|c|c|}
\hline Dye & $\begin{array}{l}\text { Output energy } \\
(\mathrm{SD})^{\mathrm{a}}\end{array}$ & $\begin{array}{l}\text { Output } \\
\text { wavelength } \\
\text { centroid }\end{array}$ \\
\hline \multicolumn{3}{|c|}{ Salts } \\
\hline Rhodamine 6G & $59.4 \mathrm{~mJ}(1.40)$ & $590 \mathrm{~nm}$ \\
\hline 4РyMPO-MePTs & $5.26(0.12)$ & 569 \\
\hline 4PyMPO-MeMs & $10.30(0.27)$ & 569 \\
\hline 4PyMPO-MeTf & $8.88(0.34)$ & 569 \\
\hline 4PyMPO-MeClO 4 & $8.30(0.39)$ & 569 \\
\hline 4PyMPO-MeBF 4 & $9.49(0.16)$ & 569 \\
\hline \multicolumn{3}{|c|}{ Zwitterions } \\
\hline 4PyMPO-SEZ & $7.43(0.24)$ & 573 \\
\hline 4PyMPO-SPrZ & $13.06(0.24)$ & 572 \\
\hline 4PyMPO-SBZ & $13.09(0.24)$ & 568 \\
\hline 4PyMPO-SPeZ & $8.51(0.25)$ & 565 \\
\hline
\end{tabular}

${ }^{\text {a }}$ standard deviation of 15 shots.

increases the energy required to neutralize it as demanded by the most likely form of the $S_{1}$ state (Figure 3), where neutralization demands that the anion move away. An alternate possibility we considered, that some extra pumping light might be absorbed by the benzene ring of the toluenesulfonate anion, and its energy transferred to the main fluorophore to increase the laser energy output, making the toluenesulfonate

Table II Energy output and wavelength of laser dyes related to 4PyMPO at $2.0 \times$ $10^{-4} \mathrm{M}$ in water when flashlamp-pumped at $20 \mathrm{KeV}$.

\begin{tabular}{|c|c|c|}
\hline Dye & $\begin{array}{l}\text { Output energy } \\
(\mathrm{SD})^{\mathrm{a}}\end{array}$ & $\begin{array}{l}\text { Output } \\
\text { wavelength } \\
\text { centroid }\end{array}$ \\
\hline \multicolumn{3}{|c|}{ Salts } \\
\hline Rhodamine $6 \mathrm{G}$ & $21.45 \mathrm{~mJ}(0.56)$ & $586 \mathrm{~nm}$ \\
\hline 4PyMPO-MePTs & $4.21(0.28)$ & 581 \\
\hline \multicolumn{3}{|c|}{ Zwitterions } \\
\hline 4PyMPO-SEZ & 6.5 & 582 \\
\hline$-\mathrm{SPrZ}$ & 7.0 & 581 \\
\hline$-\mathrm{SBZ}$ & 7.2 & 580 \\
\hline$-\mathrm{SPeZ}$ & 8.6 & 578 \\
\hline
\end{tabular}

${ }^{\text {a }}$ standard deviation of 15 shots. 


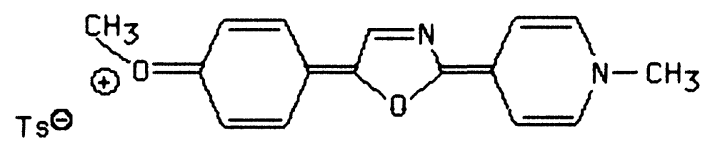

All rings and 0 -methyl in same plane

Figure 3 Proposed structure of $S_{1}$ state of 4 PyMPO-MePTs.

the most energetic certainly did not apply. The ultraviolet absorption spectrum of the toluenesulfonate, in fact, did not differ from those of the other salts.

\section{Double peaking}

Many of the dyes displayed laser output curves that were split in the middle (Figure 4), so that lasing energy appeared on either side of a dark valley located invariantly at $569 \mathrm{~nm}$. The total line width approached $12 \mathrm{~nm}$ (FWHM from the short wavelength side of the short wavelength peak to the long wavelength side of the long wavelength peak). This double peaking phenomenon is hard to explain, but it is suggested that a triplet or excited singlet absorption peak may be located at the dark valley of the lasing line, so that the system gain falls to less than unity at $569 \mathrm{~nm}$. It has been found that the molar decadic extinction coefficient of the triplet states of both rhodamine and coumarin laser dyes in their regions of lasing was $10-20 \%$ as great as the extinction coefficient for the $\mathrm{S}_{0} \rightarrow \mathrm{S}_{1}$ transition. ${ }^{1}$ If the peak were sharp, admittedly unusual for triplet manifold spectra, lasing might appear on either side of the absorption without having enough system gain to lase at the peak absorption center. That such an absorption peak does not follow their lasing line with solvent change is clear, since all four zwitterions lase in water with unsplit peaks at a redder wavelength (Table II). An attempt was made to measure the triplet state absorption spectra of the dyes ${ }^{11}$ to determine directly if a sharp absorption peak appeared at $569 \mathrm{~nm}$; no such peak was observed. However, it was also observed that the fluorescence spectrum shifted visibly, on cooling to the usual $77 \mathrm{~K}$ employed for such measurements, from the normal yellow into the blue-green. That the chromophore was modified, either physically or chemically, on going to $77 \mathrm{~K}$ was 

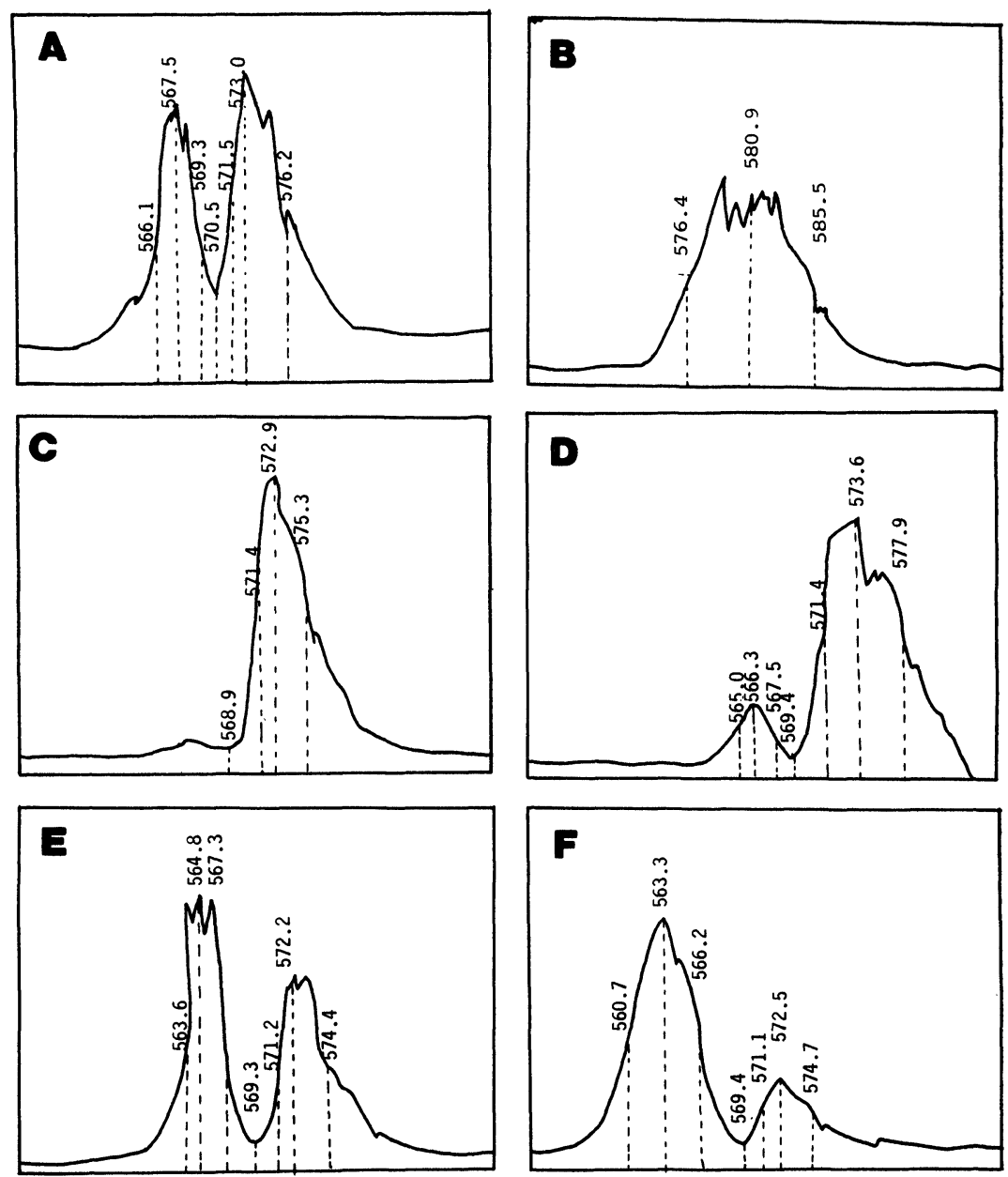

Figure 4 Oscilloscope traces of laser output of selected dyes related to 4PyMPO. A: 4PyMPO-MePTs in methanol; B: 4PyMPO-MePTs in water; C: 4PyMPO-SEZ in methanol; D: 4PyMPO-SPrZ in methanol; E: 4PyMPO-SBZ in methanol; F: 4PyMPO$\mathrm{SPeZ}$ in methanol. Peak centers and full-width half-maxima are indicated. 
evident, including the sub-structure responsible for the postulated triplet absorption.

\section{Lasing wavelength variation}

While the centroid of laser output for all the salts remained at $569 \mathrm{~nm}$ in methanol (Figure 1), the laser "lines" of the zwitterions changed wavelength in a regular order as a function of the carbon chain length in both methanol and water (Figures 1 and 2). In methanol, the "line" (both peaks together, Figure 4) moved across the dark valley invariantly located at $569 \mathrm{~nm}$ to the extent that very little energy appeared in the short wavelength peak in the sulfoethyl compound (4PyMPOSEZ), centriod $573 \mathrm{~nm}$, 2-carbon chain length; while only a small amount of energy appeared in the long wavelength peak in the sulfopentyl compound (4PyMPO-SPeZ), centroid $565 \mathrm{~nm}$, 5-carbon chain length. The peaks of the sulfopropyl compound (4PyMPOSPrZ), 3-carbon chain, and sulfobutyl compound (4PyMPO-SBZ), 4-carbon chain, appeared in between and in regular order. The same order was observed in water at generally longer wavelengths, and with about half the spread between extremes (Table II).

To explain the wavelength variation as a function of zwitterion carbon chain length, one must examine the effects of certain types of substituents on dye chromophores which cause variations in fluorescence maxima and corresponding variations in laser line location. For instance, the methoxy group of 4PyMPO causes both an increase in extinction coefficient and a red-shift of the fluorescence and lasing line relative to the unsubstituted structure $4 \mathrm{PyPO} .^{7}$ The methoxy does this via electron donation to the remainder of the fluorophore, increasing the electron density throughout the conjugated system, and also causing 4PyMPO to be the better "antenna" to absorb incoming radiation. Quaternizing the pyridyl nitrogen further increases the extinction coefficient and further red-shifts the fluorescence. One would surmise that increasing the electron density of the positively charged nitrogen would might cause a reversed effect. It is well-known that aliphatic groups are electron donators, ${ }^{12}$ and that inductive effects fall off rapidly with distance in saturated hydrocarbon chains, so that the effect of a group $-\mathrm{CH}_{2} \mathrm{R}$ is only about $2 / 5$ as great as for the group $-\mathrm{R}$; so the transmission effect of $-\mathrm{CH}_{2}$ is given as $0.41 .^{12}$ The longer zwitterion chains force more electron density onto the positive nitro- 
gen (or may be looked at as allowing less electron density drain from the nitrogen toward the negatively charged sulfonate group), explaining the blue-shift of lasing with greater chain length. This variation is especially odd because the shift occurs across an invariant null position where lasing does not occur.

The corresponding reduction in extinction coefficient was not observed; the longwave absorption peaks all appear at $405 \mathrm{~nm}$; and all the fluorescence peaks appear at $560 \mathrm{~nm}$. Perhaps this is not irreconcilable with the above explanation, since lasing is an amplification process which may exaggerate what is in reality only a small effect with respect to electron density on the fluorophore.

\section{CONCLUSIONS}

The choice of a counterion, an anion, or part of a zwitterion, for a laser dye whose fluorophore is a cation merits the experimenter's attention, since we have observed that a factor of 2.5 in additional pulse energy was available when the most energetic anion, the sulfobutyl zwitterion, was used with 4PyMPO. The commonly used anion perchlorate, in fact, gave the second weakest laser output of the nine counterions tested.

The fluorophor 4PyMPO-X exhibited a split laser line, for which we suggest, but could not demonstrate, that a triplet or excited singlet absorption is responsible.

Four sulfoalkyl zwitterions on the fluorophor 4PyMPO caused a regular shift in the centroid of the output wavelength, so that the majority of the pulse energy fell on the long or short wavelength side of a nonlasing wavelength "valley" at $569 \mathrm{~nm}$.

Impurities in one lot of spectrophotometric grade methanol which formed a chromophore under xenon flashlamp pumping which absorbed and fluoresced in the visible could be removed by distillation of the methanol from sodium borohydride. This procedure, even applied to less expensive grades of methanol, might be routinely useful for laser work.

\section{Acknowledgements}

Financial support was provided by the US Army Missle Command. We are indebted to T. G. Pavlopoulos, Naval Ocean Systems Center, San Diego, CA, USA, for attempts to 
determine triplet state absorptions, and for stimulating discussions. Computer-assisted literature searches by N. B. Rainey and technical assistance from S. J. Imbesi, P. Erndwein, L. Ruby, and A. Ghiorghis are appreciated. The help from A. Askew and E. Pilcher, in conducting the lasing tests and making the OMA pictures is greatly appreciated. The line drawings of the OMA Polaroid photographs were done in a timely manner by J. H. F. Bentley.

\section{References}

1. K.-H. Drexhage in Topics in Applied Physics 1, F. P. Schäfer, Ed. (SpringerVerlag, Berlin, 1977).

2. J. M. Kauffman, Applied Optics 19, 3431 (1980).

3. E. Neister, Phase-R Corp., private communication, 23 May 1986.

4. P. R. Hammond, IEEE J. Quantum Elect QE-15, 624 (1979).

5. T. J. Thomas and U. S. Nandi, Ind. Eng. Chem. Prod. Res. Dev. 16, 186 (1977).

6. W. R. Robinson, J. Chem. Ed. 62, 1001 (1985).

7. A. N. Fletcher, R. A. Henry, R. F. Kubin and R. A. Hollins, Optics Communs. 48, 352 (1984).

8. D. G. Ott, F. N. Hayes and V. N. Kerr, J. Am. Chem. Soc. 78, 1941 (1956).

9. R. A. Hollins, private communication, 1986.

10. J. D. Barnhurst, J. Org. Chem. 26, 4520 (1961).

11. T. G. Pavlopoulos, Spectrochimica Acta, 42A, 47 (1986).

12. D. D. Perrin, Boyd Dempsey and E. P. Serjeant, $p$ Ka Prediction for Organic Acids and Bases (Chapman and Hall, London, 1981). 